\section{PTH-117 TISSUE ENGINEERING OF VASCULARISED HUMAN LIVER ORGANOIDS: STUDY OF MORPHOLOGY PHENOTYPIC EXPRESSION AND METABOLOMICS OF TRANSITIONAL CO-CULTURES OF HEPATIC/ ENDOTHELIAL PROGENITORS}

\author{
doi:10.1136/gut.2011.239301.518
}

L J Nelson, ${ }^{1,}$ K Burgess, ${ }^{2}$ M Navarro, ${ }^{1} 0$ Tura, ${ }^{3}$ K Samuel, ${ }^{3}$ P C Hayes, ${ }^{1}$ M Turner, ${ }^{3}$ J N Plevris ${ }^{1}$ Hepatology Laboratory, The Royal Infirmary, University of Edinburgh, Edinburgh, UK; ${ }^{2}$ Scottish Metabolomics Facility, University of Glasgow, Glasgow, UK; ${ }^{3}$ MRC Centre for Regenerative Medicine, University of Edinburgh, Edinburgh, UK

Introduction Tissue architecture and hepatic cell morphology reflect the functional differentiation of the liver. Differentiated functions of hepatocytes, depend on complex, hetereotypic cell-cell/cell-matrix interactions mediated by cell adhesion molecules (CAMs) in a 3D microenvironment. Vascularisation of human hepatic tissue for therapeutic/pharmaceutical applications, requires knowledge of optimal trophic conditions to support different cell types and how these cells behave embedded in different biocompatible matrices.

Methods The authors aimed to assess morphology, metabolic functionality and phenotypic expression of human progenitor EoCs (Endothelial outgrowth Cells) vascular microvessels, cocultured with hepatic C3A cells (C3As). EoC/C3A cocultures in different media and test biomatrices: Matrigel/MaxGel/ Puramatrix (self-assembling nanofibres); and control standard 2D cultures (tissue culture plastic; TCP) were analysed following immunostaining using morphology (light/confocal microscopy); and flow cytometry. Metabolomics analysis of culture media provided a global picture of metabolic changes in response to hepatic/EoC coculture (vs reference C3A monocultures).

Results Titration in standard 2D/TCP co-culture in various media showed a ratio of 3C3A:1EoC in Lonza EGM-2 medium was optimal. Cells retained phenotypic expression of differentiation markers: (1) Hepatic: Albumin, EpCAM, E-CAD; (2) EoC: CD146, CD31, CD105, VWF; as evidenced by flow cytometry/immunostaining. Metabolomics analysis of media (from 2D/TCP co-cultures), showed modulation of key hepatic metabolic intermediaries including: (1) Urea cycle: 50\% enhancement of L-ornithine production; (2) Biosynthesis: Bile Acids: Enhanced Glycocholate; amino acid (eg, Taurine) utilisation; and Creatine production: (3) Antioxidants: co-culture ameliorated the requirement of high Glutathione antioxidant levels inherent to EoC monocultures. Test biomatrices under different 3D culture configurations, showed: (1) MaxGel sandwich culture promoted differentiated (cuboidal) morphology of C3As, but not EoCs; (2) Conversely, only EoCs overlaid on MaxGel formed differentiated (microvessel) structures; (3) Puramatrix supported 3D culture of C3As but not EoCs; (4) Matrigel supported only EoCs 3D microtubular structures.

Conclusion This study proved informative for engineering of vascularised organoids for future clinical/pharmaceutical applications. Work in progress include comprehensive metabolomics analysis, flow cytometric profiling and combinatorial analysis of cell-supportive biomatrix configurations to further optimise 3D coculture.

Competing interests None.

Keywords co-culture, endothelial progenitors, liver cell line, liver organoids. 